\title{
Evolution de Trypanosoma cruzi (Tehuantepec) chez Triatoma megista
}

\author{
par J. LAPIERRE \\ (avec la collaboration technique de M. Gilles) \\ Service de Parasitologie, Mycologie, U.E.R. Cochin-Port-Royal \\ 27, rue du Faubougr-St-Jacques, F. 75 - Paris, $14^{\circ}$
}

\begin{abstract}
Résumé
L'étude de l'évolution de $T$. cruzi (souche Tehuantepec) au niveau des différents segments du tube digestif de Triatoma megista permet de constater :

$1^{\circ}$ La persistance au niveau de l'estomac et de l'intestin jusqu'au $24^{\circ}$ jour de Trypanosomes dégradés morphologiquement, mais ayant conservé leur pouvoir infectant.

$2^{\circ}$ Au niveau de la phase rectale, l'apparition des Trypanosomes métacycliques à partir du $12^{\circ}$ jour. Avant cette date, l'inoculation à la souris du contenu du rectum ne détermine pas d'infection, ce qui confirme que les formes Crithidia ne sont pas infectantes.
\end{abstract}

\section{Summary}

Study of the life cycle of $T$. cruzi (Tehuentepec strain) in the different parts of the digestive tract of Triatoma megista shows.

$1^{\circ}$ The persistance up to the 24 th day, in the stomach and intestins, of Trypanosomes morphologically altered but having kept their infective ability.

$2^{\circ}$ In the rectal part, the formation of metacyclic trypanosomes from the 12th day. Before that date inoculation to mice of the rectum content is uneffective which confirms the fact that Crithidia forms are not infective. 


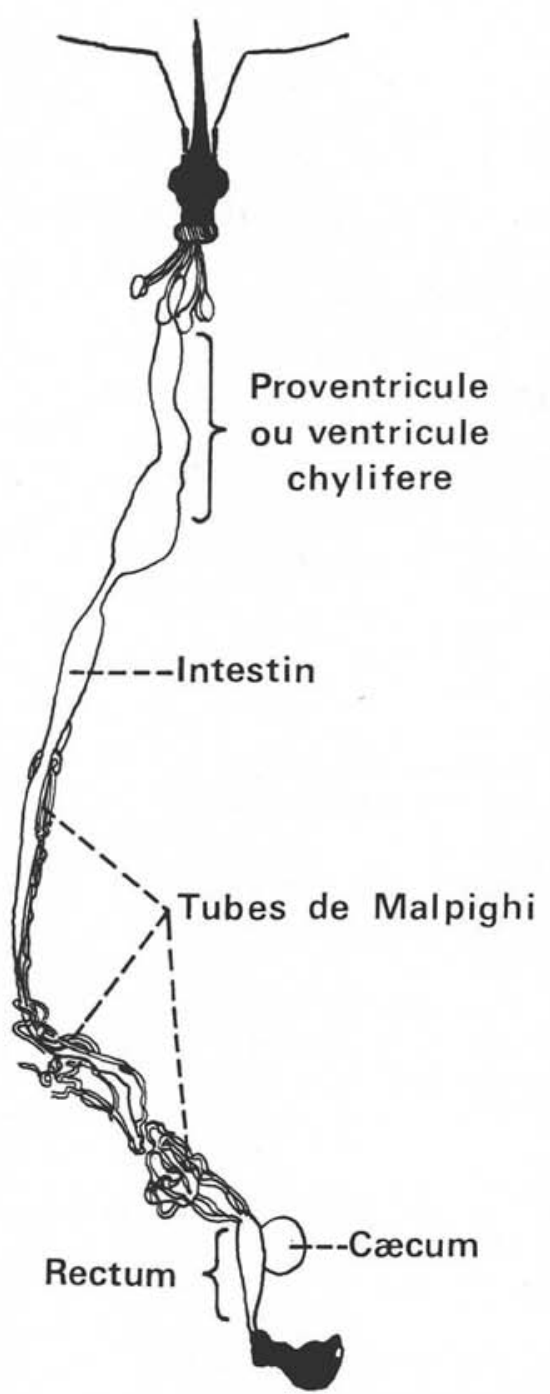

FIG. 1. - Aspect morphologique du tube digestif du Triatome. (Cf. C. Pinto, 1930)
Dans une étude expérimentale antérieure, nous avons démontré que les ponctions ou les broyats de tube digestif d'Ornithodorus moubata nourris sur souris infestées par Trypanosoma cruzi (souche Tulahuen) étaient infectants dès le lendemain du repas et le demeuraient les jours suivants avant l'apparition des formes métacycliques vers le $5^{\circ}$ jour et bien entendu par la suite. Nous pensions en trouver l'explication dans la survie des formes Trypanosomes ingérées qui conservent leur pouvoir infectant pendant au moins cinq jours bien que leur aspect morphologique soit très modifié.

Toutefois, la conformation complexe du tube digestif de l'Ornithodore ne permettait pas d'observer avec précision les phases évolutives correspondant aux différents segments du tube digestif.

Nous avons donc repris cette étude sur Triatoma megista dont l'anatomie du tube digestif (fig. 1) permet de suivre l'évolution des Trypanosomes ingérés d'abord dans le proventricule ou ventricule chylifère (phase stomacale), puis ultérieurement dans les différents segments de l'intestin (phases intestinales et rectales).

\section{Protocole expérimental.}

Un lot de 25 Triatomes a été gorgé sur des souris présentant une riche infection (50 $\mathrm{Tr}$ par champ microscopique au grossissement 250) à Trypanosoma cruzi (souche Tehuantepec). Nous avons employé cette souche de préférence à la souche Tulahuen utilisée antérieurement en raison des infections plus importantes qu'elle détermine chez la souris.

Les Triatomes infectés ont été conservés dans une enceinte à $25^{\circ}$. Chaque jour à partir du lendemain du repas infestant et jusqu'au $24^{\circ}$ jour, un ou plusieurs Triatomes ont été sacrifiés et leur tube digestif disséqué afin de séparer le proventricule, l'intestin et le rectum (fig. 1). 
$1^{\circ}$ A des fins d'études morphologiques, des frottis ont été réalisés avec le contenu du proventricule, avec celui de l'intestin après léger délayage dans le sérum physiologique et enfin à partir des déjections pures émises par l'insecte ou recueillies avant de le sacrifier. La coloration des lames a été faite par la technique du May-GrunwaldGiemsa avec toutefois une fixation à frais par le Lugol double pour les frottis de déjection.

$2^{\circ}$ Enfin, des souris neuves ont été inoculées par voie intrapéritonéale sous couvert de Pénicilline (1.000 U) avec le contenu du proventricule, de l'intestin et les déjections.

\section{A) Etude morphologigue.}

\section{$1^{\circ}$ Phase stomacale.}

$L^{\prime}$ observation directe du contenu du ventricule à la $24^{\circ}$ heure permet de constater qu'une bonne partie des Trypanosomes restent mobiles. A la $48^{\circ}$ heure, quelques Trypanosomes ont conservé une faible mobilité et beaucoup entrent en voie de lyse. A partir du $3^{\circ}$ jour, l'observation directe ne permet plus de reconnaître les parasites. Le caillot sanguin subit d'abord une régression de volume rapide jusqu'au $3^{\circ}$ jour, puis ensuite est lentement digéré jusque vers le $20-25^{\circ}$ jour, en conservant toutefois toujours un aspect rougeâtre qui tranche avec la coloration noirâtre du contenu intestinal.

Sur frottis coloré, l'étude morphologique permet de constater dès le $1^{\text {er }}$ jour (fig. 2,b) une réduction de taille des formes Trypanosomes observées avec tendance à la disparition du flagelle libre.

A la $48^{\circ}$ heure (fig. 2, c), on observe une grande majorité de formes arrondies avec persistance du flagelle enroulé à l'intérieur du protoplasme.

Du $3^{\circ}$ au $6^{e}$ jour (fig. 2, d), ces mêmes formes arrondies avec conservation du flagelle enroulé continuent à être présentes. Dans certains cas, cependant, le flagelle n'est plus observable et il est alors difficile de dire si sa disparition correspond à un artefact ou à une évolution vers la forme Leishmania. Au milieu de cet ensemble de formes arrondies, on peut néanmoins observer des formes plus ou moins allongées dont certaines ont conservé l'aspect Trypanosome caractéristique avec toutefois une réduction de taille.

Sur les frottis effectués jusqu'au $24^{\circ}$ jour, on observe pratiquement toujours le même aspect.

L'existence de ces éléments arrondis, d'aspect «leishmaniforme», a donné lieu à des interprétations diverses suivant les auteurs. Minchin (1914) pense que lors de la phase stomacale des leishmanias pourraient dériver directement des formes Trypanosomes du sang, mais que ce phénomène constitue une phase inconstante, la partie essentielle stomacale étant la transformation des parasites sanguicoles en Crithidia qui correspondent à la forme larvaire ancestrale des hémoflagellés. Pour E. Brumpt (1927), les aspects arrondis qu'il observe en grand nombre dans le ventricule chylifère sont bien des formes Leishmania. Pour E. Diaz (1934), les formes flagellés observées 

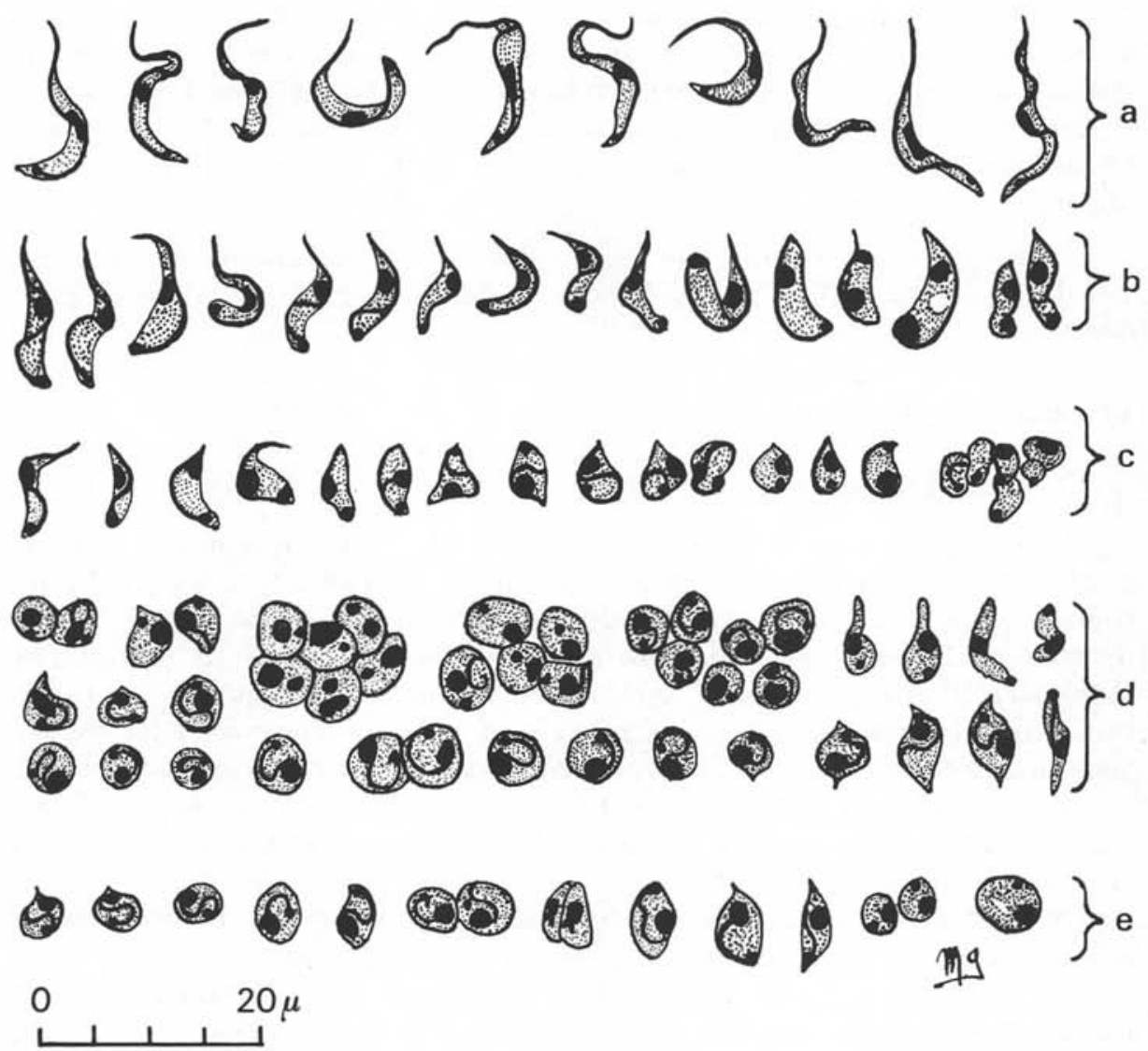

FIG. 2. - Formes évolutives de T. cruzi (Tehuantepec) dans le caillot sanguin du proventricule (ventricule chylifère du Triatome): $a$ ) formes sanguicoles ingérées au moment du ropas infectant, b) formes du $1^{\text {er }}$ jour, $c$ ) du $2^{\circ}$ jour, $d$ ) du $3^{\circ}$ au $6^{\circ}$ jour, e) au-delà du $24^{e}$ jour (formes rares). Noter l'évolution morphologique des trypanosomes et leur transformation dès le $3^{e}$ jour en formes arrondies, immobiles, ne s'observant plus à l'examen direct, mais seulement sur frottis colorés

dans l'estomac correspondent aux types suivants: Trypanosomes non modifiés ou légèrement altérés, Trypanosomes de transition évoluant vers la forme Crithidia, Crithidia, formes arrondies avec ou sans flagelles libres, parasites irréguliers plus ou moins altérés. Il déclare n'avoir pu retrouver les grandes masses de Leishmania signalées par E. Brumpt.

Nos observations sont très proches de celles de Diaz et nous sommes personnellement tentés de considérer que les aspects arrondis «leishmaniformes que nous avons observé (fig. 2, c, d,e), correspondent plutôt à des formes trypanosomes morphologiquement altérés qu'à de véritables formes Leishmania. Deux arguments sont à retenir : 

enroulé ;

- la possibilité de mettre en évidence chez la plupart d'entre elles un flagelle

— en recueillant ces «formes flagellées» caractéristiques dans l'estomac de Triatomes, six jours après le repas infectant et en les faisant incuber à $37^{\circ}$ pendant trois heures dans du sérum de souris, il nous a été possible d'observer des modifications morphologiques tendant à faire évoluer par allongement et libération du flagelle, ces formes arrondies pseudo-leishmaniennes, vers un très net aspect Trypanosome (fig. 3).

Par contre, à l'inverse de Minchin et de Diaz, nous n'avons pas, lors de la phase stomacale suivie du $1^{\text {er }}$ au $24^{\circ}$ jour, observé de formes Crithidia caractéristiques alors
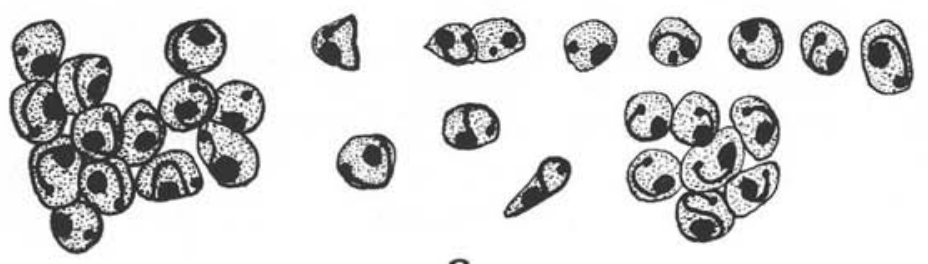

a

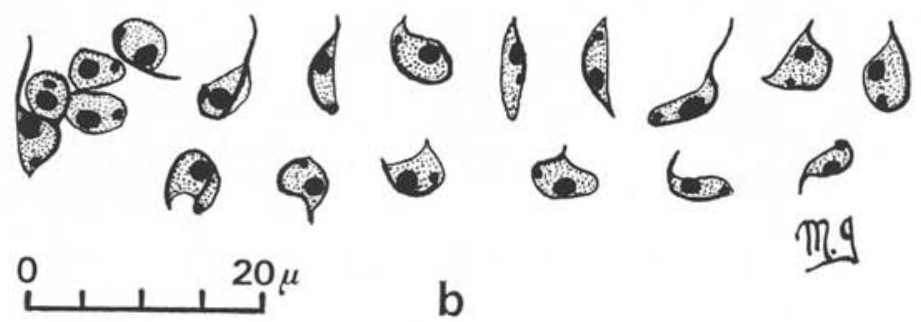

FIG. 3. - $-a$ ) Parasites du caillot sanguin du proventricule au $6^{e}$ jour après le repas infectant. $b$ ) Transformations morphologiques des mêmes formes après une incubation de 3 heures à $37^{\circ}$ dans le sérum de souris neuve

que nous les avons trouvées en grand nombre dans l'intestin dès la $48^{\circ}$ heure (voir ci-après). A moins qu'il ne s'agisse d'une caractéristique particulière de notre souche (Tehuantepec), nous ne pensons pas que le biotope stomacal soit favorable à une évolution et à une multiplication du parasite vers et sous la forme Crithidia.

\section{$2^{\circ}$ Phase intestinale.}

Les Flagellés qui passent dans l'intestin pour y poursuivre leur évolution revêtent très rapidement la forme Crithidia, sous laquelle ils vont se multiplier activement par division binaire. 

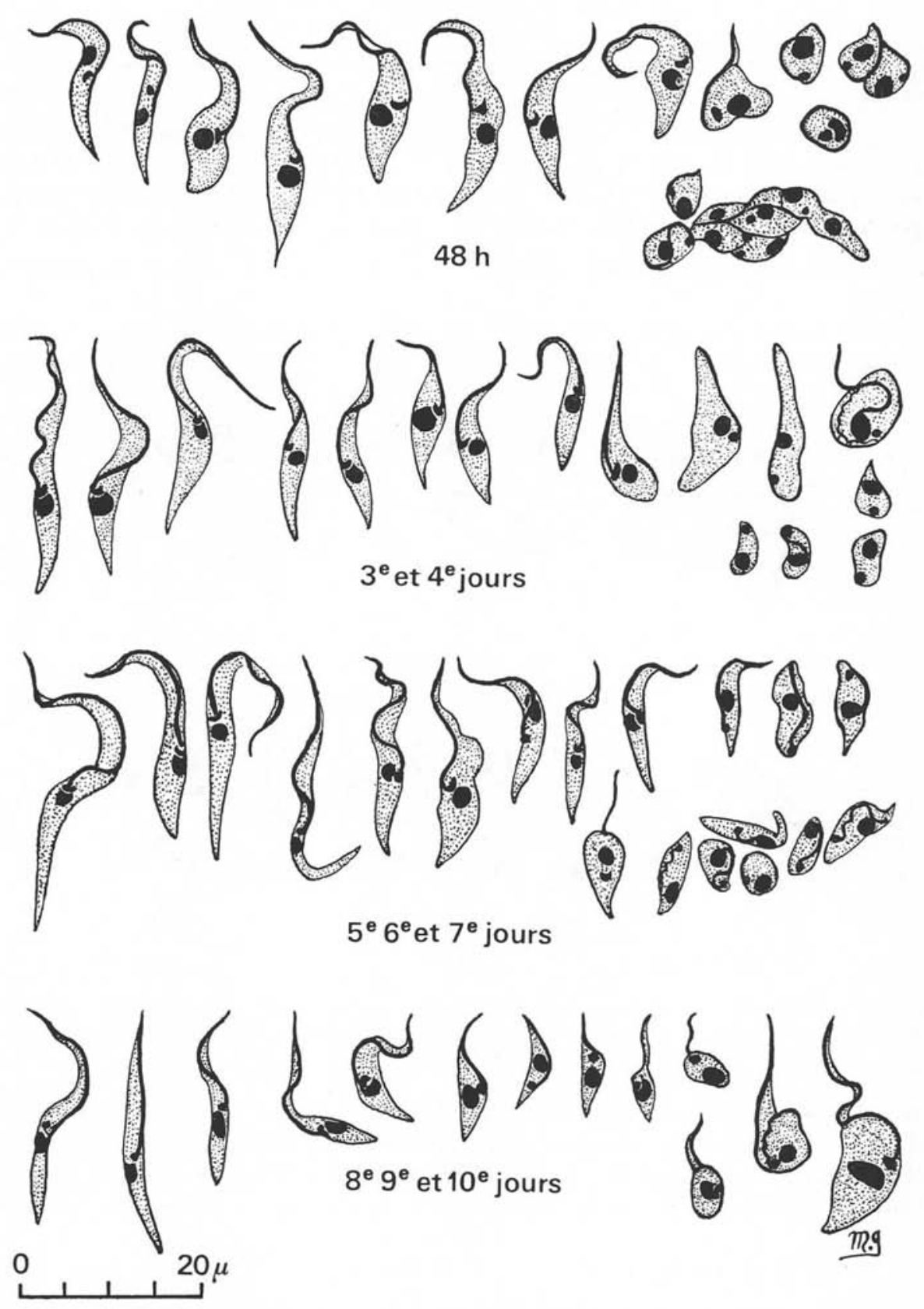

FIG. 4. - Formes évolutives de $T$. cruzi (Tehuantepec) dans l'intestin du Triatome du $2^{e}$ au $10^{\circ}$ jour après le repas infectant. Passé ce délai, la vacuité de l'intestin gêne considérablement la réalısation de frottis sur lesquels, par ailleurs, les formes observées sont très rares 
L'examen direct du contenu intestinal et l'étude des frottis colorés (fig. 4) permettent de constater une prolifération intense dès le $2^{\circ}$ et $3^{\circ}$ jour, donnant des formes de dimensions très variables. L'existence de ces formes dans l'intestin va se maintenir durant toute la vie de l'insecte. Certes, vers le $10^{\circ}$ jour, la vacuité de l'intestin gêne la réalisation des frottis sur lesquels les flagellés observés sont rares, mais il suffit d'un repas de sang (non infecté) sur souris saine pour voir à nouveau se produire une prolifération intense.

A côté de ces formes Crithidia caractéristiques de la phase intestinale, on peut retrouver sur les frottis pendant les huit premiers jours ces formes arrondies «pseudoleishmaniennes » précédemment décrites et que nous avons assimilées à des Trypanosomes altérés. L'existence de ces formes doit d'ailleurs persister au-delà de huit jours puisque, comme nous le verrons ultérieurement, l'inoculation du contenu de l'intestin détermine une infection.

Par contre, nous n'avons pas retrouvé dans l'intestin de formes Trypanosomes sanguicoles caractéristiques telles que Diaz en particulier a pu les observer.

Enfin, nous n'avons jamais observé de formes Trypanosomes métacycliques correspondant à l'aboutissement du cycle qui ne se retrouvent que dans la phase rectale.
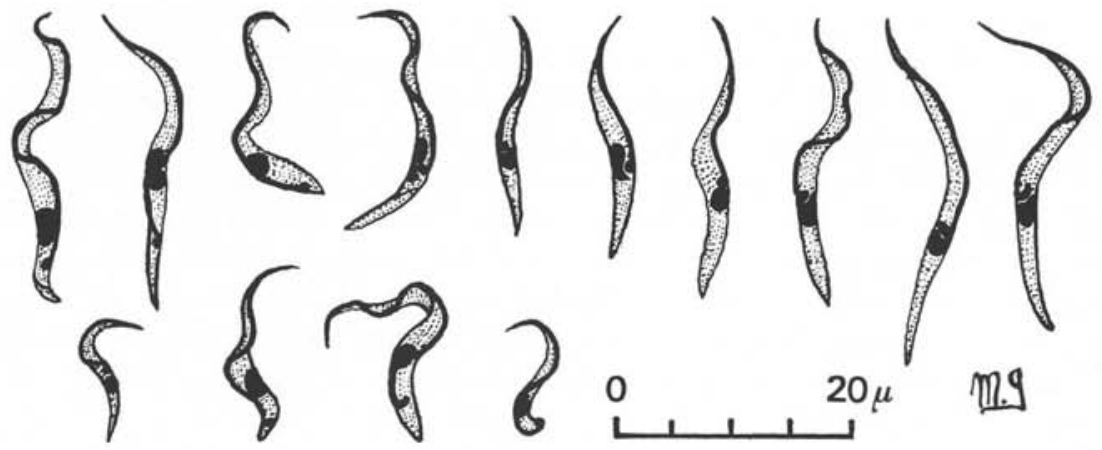

FIG. 5. - Formes évolutives de $T$. cruzi (Tehuantepec) des déjections du Triatome 12 jours après le repas infectant

\section{$3^{\circ}$ Phase rectale.}

Dans la partie postérieure du tube digestif, on trouve une accumulation de flagellés allant de la forme Crithidia aux formes métacycliques de Trypanosomes qui constituent la forme de multiplication caractéristique de la phase rectale, avec bien entendu tous les stades de transition entre l'un et l'autre. Nous n'avons vu apparaître les formes métacycliques caractéristiques que le $12^{\circ}$ jour après le repas infectant alors que Chagas constatait leur existence dès le $6^{\circ}$ jour et Diaz vers le $6^{\mathrm{e}}-7^{\mathrm{e}}$ jour. Il est possible que ce retard soit dû à la biologie particulière de la souche ou aux conditions de conservation des Triatomes (température de $25^{\circ}$ ). 


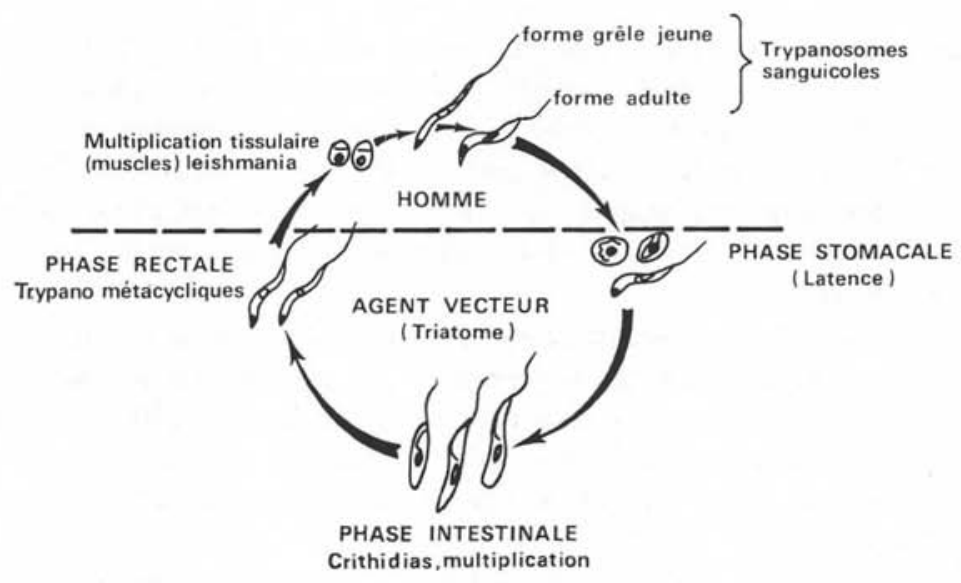

FIG. 6. - Cycle évolutif de Trypanosoma cruzi

B) Inoculation à la souris du contenu des différents segments du tube digestif.

a) Inoculation du CAIllot DU PROVEnTRicule.

L'inoculation du caillot du proventricule prélevé chez le Triatome du $1^{\text {er }}$ au $24^{\circ}$ jour après le repas infectant détermine d'une façon constante une infection mortelle chez la souris. Ceci se comprend parfaitement pour les prélèvements effectués jusqu'au troisième jour puisqu'il est facile d'observer des Trypanosomes sanguicoles ayant conservé toute leur vitalité et leurs caractéristiques morphologiques. Pour la suite, en raison du maintien du pouvoir infectant, il faut admettre que les formes arrondies « pseudo-leishmaniennes » observées ne sont pas des formes leishmania, mais en réalité des Trypanosomes de survie, morphologiquement dégradés. La présence d'un flagellé enroulé à l'intérieur du cytoplasme représente par ailleurs un argument morphologique qui, joint au pouvoir infectant, nous permet d'assurer qu'il s'agit bien de stade * Trypanosoma » du $T$. cruzi.

\section{b) INOculation du CONTENU DE L'INTESTin.}

Le contenu de l'intestin prélevé du $1^{\text {er }}$ au $24^{\circ}$ jour après le repas infectant et inoculé à des souris neuves entraîne constamment une infection mortelle chez les animaux inoculés. Etant donné l'absence de Trypanosomes métacycliques dans la phase intestinale, il faut donc là encore expliquer le pouvoir infectant par la survie de Trypanosomes sous cette forme dégradée précédemment décrite.

c) Inoculation des déjections du Triatome.

Elle ne détermine une infection mortelle chez la souris qu'à partir du $12^{\circ}$ jour, correspondant d'ailleurs à l'observation des Trypanosomes métacycliques lors de l'observation microscopique directe. 
INOCULATION A LA SOURIS DU CONTENU DES DIFFÉRENTS SEGMENTS DU TUBE DIGESTIF DE TRIATOME INFECTÉS

\begin{tabular}{|c|c|c|c|}
\hline 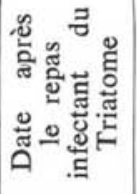 & $\begin{array}{l}\text { Caillot stomacal } \\
\text { (phase stomacale) }\end{array}$ & $\begin{array}{c}\text { Intestin } \\
\text { (phase intestinale) }\end{array}$ & $\begin{array}{c}\text { Déjections } \\
\text { (phase rectale) }\end{array}$ \\
\hline $24 \mathrm{~h}$. & $+12^{\circ} \mathrm{j}$. Décès $_{20 \mathrm{Tr} / \times} 34^{\circ} \mathrm{j}$. & - & 一 \\
\hline $48 \mathrm{~h}$. & $+12^{\circ} \underset{20 \mathrm{jr} / \text { Décès }^{\circ}}{35^{\circ} \mathrm{j} .}$ & $\begin{array}{c}+16^{\circ} \text { j. Décès } 35^{\circ} \text { j. } \\
100 \mathrm{Tr} / \times\end{array}$ & - \\
\hline $3 \mathrm{j}$. & $+11^{\mathrm{e}} \mathrm{j}$. Décès $33 \& 35^{\mathrm{e}} \mathrm{j}$ & $+1^{\circ} \mathrm{j} .{ }_{50 \mathrm{Tr} / \times^{\text {Décès }} 35 / 36^{\circ} \mathrm{j}}$ & - \\
\hline $4 \mathrm{j}$. & $\begin{array}{c}+15^{\circ} \mathrm{j} . \text { Décès } 31^{\circ} \mathrm{j} . \\
100 \mathrm{Tr} / \times\end{array}$ & $\begin{array}{c}+12^{\circ} \text { j. Décès } 31 \& 53^{\circ} \mathrm{j} . \\
100 \mathrm{Tr} / \times\end{array}$ & - \\
\hline $5 \mathrm{j}$. & $\begin{array}{c}20^{\circ} \mathrm{j} \text {. Décès } 28^{\circ} \mathrm{j} \text {. } \\
30 \mathrm{Tr} / \times\end{array}$ & $+14^{\circ} \mathrm{j}$. Décès $29^{\circ} \mathrm{j}$. & - \\
\hline $6 \mathrm{j}$. & $+11^{\circ}$ j. Décès $22 \& 32^{\circ} \mathrm{j}$. & $\begin{array}{c}+18^{\circ} \text { j. Décès } 49^{\circ} \text { j. } \\
100 \mathrm{Tr} / \times\end{array}$ & - \\
\hline $7 \mathrm{j}$. & $\begin{array}{c}+13^{\mathrm{e}} \text { j. Décès } 40 \text { \& } 43^{\mathrm{e}} \mathrm{j} . \\
20 \mathrm{Tr} / \times\end{array}$ & $\begin{array}{l}+10^{\circ} \text { j. Décès } 32 \& 43^{\circ} \mathrm{j} . \\
30 \mathrm{Tr} / \times\end{array}$ & - \\
\hline $8 \mathrm{j}$. & $\begin{array}{c}+13^{\circ} \mathrm{j} . \text { Décès } 45^{\circ} \mathrm{j} . \\
100 \mathrm{Tr} / \times\end{array}$ & $\begin{array}{c}+15^{\circ} \mathrm{j} . \text { Décès } 42^{\circ} \mathrm{j} . \\
100 \mathrm{Tr} / \times\end{array}$ & - \\
\hline $10 \mathrm{j}$. & $\begin{array}{c}+17^{\circ} \text { j. Décès } 46^{e} \mathrm{j} . \\
40 \mathrm{Tr} / \times\end{array}$ & $\begin{array}{c}+17^{\circ} \text { j. Décès } 27 \& 30^{\circ} \mathrm{j} . \\
100 \mathrm{Tr} / \times\end{array}$ & - \\
\hline $12 \mathrm{j}$. & $+16^{\circ} \mathrm{j}$. Décès $_{30 \mathrm{Tr} / \times} 31^{\circ} \mathrm{j}$. & $\begin{array}{c}+19^{\circ} \mathrm{j} . \text { Décès } 42^{\circ} \mathrm{j} . \\
100 \mathrm{Tr} / \times\end{array}$ & $+14^{\circ} \mathrm{j}$. Décès $31^{\circ} \mathrm{j}$. \\
\hline $15 \mathrm{j}$. & $+14^{\circ} \mathrm{j}$. Décès $34^{e} \mathrm{j}$. & $\begin{array}{c}+19^{\circ} \mathrm{j} \text {. Décès } 46^{e} \mathrm{j} . \\
100 \mathrm{Tr} / \times\end{array}$ & $+15^{\circ}$ j. Décès $30^{\circ} \mathrm{j}$. \\
\hline $24 \mathrm{j}$. & $\begin{array}{c}+15^{\mathrm{e}} \mathrm{j} . \text { Décès } 46^{\circ} \mathrm{j} . \\
30 \mathrm{Tr} / \times\end{array}$ & $\begin{array}{c}+18^{\circ} \text { j. Décès } 43^{\circ} \text { j. } \\
100 \mathrm{Tr} / \times\end{array}$ & $+15^{\circ}$ j. Décès $27^{\circ} \mathrm{j}$. \\
\hline
\end{tabular}

\section{Bibliographie}

BRUMPT (E.), 1912. - Le Trypanosoma cruzi évolue chez Conorhinus megistus, Cimex lectularius, Cimex boueti et Ornithodorus moubata. Cycle évolutif de ce parasite. Bull. Soc. Path. Exot., V, p. 360,

-, 1913. - Evolution de Trypanosoma lewisi, duttoni, nabiasi, blanchardi, chez les puces et les punaises. Transmission par les déjections. Comparaison avec T. cruzi. Bull. Soc. Path. Exot., VI, p. 167-171. 
-, 1913. - Immunité partielle dans les infections à Trypanosoma cruzi. Transmission de ce Trypanosome par Cimex rotundatus. Rôle régulateur des hôtes intermédiaires. Passage à travers la peau. Bull. Soc. Path. Exot., VI, p, 172-176,

-, 1936. Précis de Parasitologie, 5e édition, Masson et $\mathrm{C}^{1 \mathrm{e}}$, t. I et t. II, $2.139 \mathrm{pp}$.

- et PIRAJa DA Silva, 1912. - Existence du Schizotrypanum cruzi Chagas, 1909 à Bahia (Matta de Sao Joao). Biologie du «Conorhinus megistus, Bull. Soc. Path. Exot. V, p. 22-26.

- et Gonzalez-Lugo, 1913. - Présentation d’un Réduvidé du Vénézuéla, le Rhodnius prolixus, chez lequel évolue Trypanosoma cruzi. Bull. Soc. Path. Exot., VI, p. $382-383$.

-, Mazotri (L.) et Brumpt (L. C.), 1939. - Enquêtes épidémiologiques sur la maladie de Chagas au Mexique. Réduvidés vecteurs. Animaux réservoirs de virus. Cas humains. Ann. Parasit., XVII, n 4, p. 299-312.

Chagas (C.), 1909. - Nova tripanozomiaze humana. Estudos sobre a morfolojia e o ciclo evolutivo do Schizotrypanum cruzi n. gen. n. sp., ajente étiologico de nova entidade morbida do homen. Mem. Inst. Oswaldo Cruz, I, p. 159-218.

-, 1911. - Nova entidade morbida do homen. Rezumo geral de estudos etiolojicos e clinicos. Mem. Inst. Oswaldo Cruz, III, p. 219-275.

-, 1911. - Le cycle de Schizotrypanum cruzi chez l'homme et les animaux de laboratoire. Bull. Soc. Path. Exot., IV, p. 467-471,

-, 1927. - Quelques aspects évolutifs du Trypanosoma cruzi dans l'insecte transmetteur. C.R. soc. de Biol., XCVII, p. 829.

Delanoe (M.) et $\mathrm{M}^{\mathrm{me}}$, 1912. - A propos du Schizotrypanum cruzi. Bull. Soc. Path. Exot: V, p. 599.

Dins (E.), 1934. - Estudos sobre o Schizotrypanum cruzi. Mem. Inst. Oswaldo Cruz, XXVIII, 1-110.

Dias-Ungria (C.), 1965. - Transmission del Trypanosoma cruzi en los vertebrados. Rev. Iber. Parasit., Vol. XXV (3-4), p. 321-356.

-, 1966. - Transmission du Trypanosoma cruzi chez les mammifères. Ann. Parasit., XLI, $\mathrm{n}^{\circ} 6$, p. 549-571.

DunN (L. H.), 1934. - Attempts to transmit Trypanosoma cruzi Chagas with ticks of the genus Ornithodoros. Amer. J. Trop. Med., XIV, ${ }^{\circ}$ 3, p. 283:

ElKeles (G.), 1944. - Investigaciones sobre la evolution del Trypanosoma cruzi, specialmente sobre la genesis de la forma Trypanosomica. Bol. Acad. Nac. Ciencias, XXXVI, p. $330-408$.

Galliard (H.), 1929. - Remarques sur la culture de Trypanosomia cruzi, Chagas. Ann. Parasit., VII, $\mathrm{n}^{\circ}$ 5, p. 367-376.

—, LAPIERRe (J.), Rousset (J. J.), 1959. - Atténuation de l'infection à Trypanosoma cruzi chez la souris blanche par différentes souches de Borrelia. Bull. Soc. Path. Exot. $52, \mathrm{n}^{\circ} 3$, p. $272-276$.

Jadin (J.), Chantraine (J.), Pierreux (G.) et van Braeckel (G.), 1960. - Etude d'une souche congolaise de Trypanosoma lewisi. Ann. Soc. Belge, Méd. Trop., VL, $\mathrm{n}^{\circ}$ 6, p. 907-914. 
MaYer (M.), 1918. - Ueber den Dauerparasitismus von Schizotrypanum cruzi bei Ornithodorus moubata. Arch. f. Schi. Trop. Hyg, XXII, p, 158-160,

- et da Rocha Lima (H.), 1914. - Zum Verhalten von Schizotrypanum cruzi in Warmbliitern und Artropoden. Arch. f. Sch. Trop. Hyg, XVIII, p, 101,

Mazotti (L.) et Osorio (M. T.), 1943. - Experimentos de transmission de Trypanosoma cruzi en cuatro especies de «Ornithodoros». Rev. Inst. Salub. y Enferm. Trop,, IV, p. 163-165.

MUNIZ (J.), 1927. - Quelques formes intéressantes trouvées dans les cultures de Trypanosoma cruzi. C.R. Soc. Biol., XCVII, p, 821,

Nerva (A.), 1913. - Transmissao do Trypanosoma cruzi pelo Rhipicephalus sanguineus. Brazil medico, $\mathrm{n}^{\circ} 46$, p. 498.

Nino (F. L.), 1929. - Contribucion al estudio de la Enfermedad de Chagas o Trypanosomiasis Americana en la Républica Argentina. Mision de Estudios de Patologia Regional Argentina, Buenos Aires, 1929, 237 pp.

Noller (W.), 1920. - Neue Forschungen auf dem gebiete der Trypanosomenzuchtung. Arch. f. Schiffund Trop. Hyg., XXIV, p. 168,

PICK (F.), 1957. - Sur un nouveau milieu au sang permettant la transmission rapide du Trypanosoma cruzi (formes sanguines et métacycliques) en formes leishmaniennes. Bull. Soc. Path. Exot., T. 50, n ${ }^{\circ}$ 2, p, 217-219,

Pinto (C.), 1931. - Arthropodes parasitos e transmissores de Doenças. Brasil, Rio de Janeiro, I et II, p. 845 .

RodHain (J.), 1942. - Au sujet du développement intracellulaire de Trypanosoma lewisi chez Ornithododorus moubata. Acta, Biol., Belge, IV, p. 413.

- et Brutsaert (P.), 1935. - L'évolution des Trypanosoma lewisi et Trypanosoma cruzi chez Melophagus ovinus. C.R. Soc. Biol., CXVIII, p, 1228,

Torres (C. M.), 1922. - Cultura do Schizotrypanum cruzi Chagas, 1920, en meio liquido. Influencia da concentraçao dos ions de hydrogenio sobre a cultura. Verificaçao precoce do Schizotrypanum no sangre. Brasil Medico, p. 317.

URIBE (C.), 1926. - On the biology and life-history of Rhodnius prolixus Stahl. J. Parasit. Urbane, XIII, p. 129-136.

Vergani (F.), 1952. - Estudio sobre la veccion de Trypanosomas por medio de dipteros no vulnerentes. Bol. Inv. Vet., IV, 20, p. 657-672,

Woop (F.S.), 1943. - Addition notes on the persistence of Trypanosoma cruzi in dead insect vectors. Bull. Southern California Academy of Sciences, XLII, Part. 3. 